\title{
ON ALMOST SEMI-FREDHOLM LINEAR RELATIONS IN NORMED SPACES
}

\author{
T. ÁLVAREZ \\ Department of Mathematics, University of Oviedo, 33007 Oviedo, Spain \\ e-mail: seco@pinon.ccu.uniovi.es
}

(Received 17 May, 2004; accepted 11 October, 2004)

\begin{abstract}
We give characterisations of the linear relations that are injective and open (respectively, almost open with dense range) in terms of the stability of the nullity (respectively, of the deficiency). Results of Mbekhta about bounded operators in Banach spaces (J. Operator Theory 35 (1996), 191-201) are covered.
\end{abstract}

2000 Mathematics Subject Classification. 47A06.

1. Introduction and preliminaries. We follow the notation and terminology of the book [5]: $X$ and $Y$ are normed spaces, $B_{X}$ the closed unit ball of $X, X^{\prime}$ the dual space of $X$. If $M \subset X, N \subset X^{\prime}$ are subspaces, then $M^{\perp}:=\left\{x^{\prime} \in X^{\prime}: x^{\prime}(m)=0\right.$ for all $\left.m \in M\right\}$, $N^{\top}:=\left\{x \in X: x^{\prime}(x)=0\right.$ for all $\left.x^{\prime} \in N\right\}$.

A linear relation or multivalued linear operator $T: X \rightarrow Y$ is a mapping from a subspace $D(T) \subset X$, called the domain of $T$, into $P(Y) \backslash\{\emptyset\}$ (the collection of nonempty subsets of $Y$ ) such that $T\left(\alpha x_{1}+\beta x_{2}\right)=\alpha T\left(x_{1}\right)+\beta T\left(x_{2}\right)$, for all nonzero scalars $\alpha, \beta$ and $x_{1}, x_{2} \in D(T)$. The class of such relations $T$ is denoted by $L R(X, Y)$. If $T$ maps the points of its domain to singletons, then $T$ is said to be a single valued linear relation or simply operator.

The graph $G(T)$ of $T \in L R(X, Y)$ is $G(T):=\{(x, y): x \in D(T), y \in T x\}$. The closure of $T$, denoted by $\bar{T}$, is defined by $G(\bar{T}):=\overline{G(T)}$. Let $M$ be a subspace of $D(T)$. Then the restriction $\left.T\right|_{M}$ is defined in terms of its graph by

$$
G\left(\left.T\right|_{M}\right):=\{(m, y): m \in M, y \in T m\} .
$$

The inverse of $T$ is the linear relation $T^{-1}$ defined by $G\left(T^{-1}\right):=\{(y, x) \in Y \times X$ : $(x, y) \in G(T)\}$. If $T^{-1}$ is single valued, then $T$ is called injective; that is, $T$ is injective if and only if its null space $N(T):=T^{-1}(0)=\{0\}$, and $T$ is said to be surjective if its range $R(T):=T(D(T))=Y$. The nullity and the deficiency of $T \in L R(X, Y)$ are defined respectively by $\alpha(T):=\operatorname{dim} N(T)$ and $\bar{\beta}(T):=\operatorname{dim} Y / \overline{R(T)}:=\operatorname{codim} \overline{R(T)}$. We also write $\beta(T):=\operatorname{dim} Y / R(T):=\operatorname{codim} R(T)$. If either $\alpha(T)$ or $\bar{\beta}(T)$ (respectively, $\alpha(T)$ or $\beta(T)$ ) are finite, then we define the reduced index (respectively, the index) of $T$ by $\overline{1}(T):=\alpha(T)-\bar{\beta}(T)$ (respectively, $i(T):=\alpha(T)-\beta(T)$ ).

The conjugate or adjoint $T^{\prime}$ of $T$ is defined by $G\left(T^{\prime}\right):=G\left(-T^{-1}\right)^{\perp} \subset Y^{\prime} \times X^{\prime}$ where $\left\langle(y, x),\left(y^{\prime}, x^{\prime}\right)\right\rangle:=\left\langle x, x^{\prime}\right\rangle+\left\langle y, y^{\prime}\right\rangle$. This means that $\left(y^{\prime}, x^{\prime}\right) \in G\left(T^{\prime}\right)$ if and only if $y^{\prime}(y)=x^{\prime}(x)$ for all $(x, y) \in G(T)$. For a given $T \in L R(X, Y)$ let $Q_{T}$ denote the 
quotient map from $Y$ onto $Y / \overline{T(0)}$. Clearly $Q_{T} T$ is a single valued and the norm of $T$ is defined by $\|T\|:=\left\|Q_{T} T\right\|$.

A linear relation $T \in L R(X, Y)$ is said to be closed if its graph is a closed subspace, continuous if for each neighbourhood $V$ in $R(T), T^{-1}(V)$ is a neighbourhood in $D(T)$ (equivalently $\|T\|<\infty$ ), open if $T^{-1}$ is continuous (equivalently $0<\gamma(T):=\sup \{\lambda$ : $\|T x\| \geq \lambda d(x, N(T))$ for $x \in D(T)\}$, almost open if $\overline{T B_{X}} \supset \lambda B_{R(T)}$ for some $\lambda>0, \phi_{+}$ if it has finite dimensional null space and closed range and $\phi_{-}$if its range is closed and finite codimensional. Continuous everywhere defined linear relations are referred to as bounded linear relations. The class of such relations is denoted by $B(X, Y)$.

Linear relations were introduced into Functional Analysis by J. von Neumann [10] motivated by the need of considering conjugates of non-densely defined linear differential operators which are considered by several authors (see, for example, [3] and [4]). Problems in optimisation and control also lead to the study of linear relations; (see [1] and [2] among others). Others works on multivalued linear operators include the treatise of partial differential relations by Gromov [7] and the application of multivalued methods to the solution of differential equations by Favini and Yagi [6].

We note that the definition of linear relation "open" (respectively, "almost open") is formally identical with Harte's "relatively open" (respectively, "relatively almost open") of an operator (Harte [8, 3.4.1 and 3.5.5]). Thus, with our terminology and according to [8, 6.10.1], we say that a bounded operator $T: X \rightarrow Y$ is almost upper semi-Fredholm if $T$ is open with finite dimensional null space and $T$ is called almost lower semi-Fredholm if it is almost open and $\operatorname{dim} Y / \overline{R(T)}<\infty$.

These notions can be generalised naturally to arbitrary multivalued linear operators as follows.

Definition 1. A linear relation $T \in L R(X, Y)$ is called

(i) almost upper semi-Fredholm if it is open and $\operatorname{dim} N(T)<\infty$,

(ii) almost lower semi-Fredholm if it is almost open and $\operatorname{dim} Y / \overline{R(T)}<\infty$.

The corresponding classes of linear relations will be abbreviated $\operatorname{AUSF}(X, Y)$ and $A \operatorname{LSF}(X, Y)$ respectively.

The following Proposition is elementary and helps to understand the main results (Theorems 7 and 11 below).

Proposition 2. Let $X$ and $Y$ be Banach spaces and let $T \in L R(X, Y)$ be closed.

(i) $T$ is injective and open if and only if $T$ is injective and has closed range.

(ii) $T$ is AUSF if and only if $T$ is $\phi_{+}$.

(iii) $T$ is almost open with dense range if and only if $T$ is surjective.

(iv) $T$ is ALSF if and only if $T$ is $\phi_{-}$.

Proof. It is sufficient to note that a linear relation is almost open if and only if its conjugate relation is open [5, III.5.2]. From the Closed Graph and Open Mapping Theorems for multivalued linear operators (see [5, III.5.3 and III.5.4]), it follows that for closed linear relations between Banach spaces $T$ is open $\Leftrightarrow T^{\prime}$ is open $\Leftrightarrow R(T)$ is closed $\Leftrightarrow R\left(T^{\prime}\right)$ is closed.

In the context of bounded operators in Banach spaces, Mbekhta proves the following characterisation results.

Theorem $3[9,2.6]$. Let $T: X \rightarrow Y$ be a bounded operator, where $X$ and $Y$ are Banach spaces. The following conditions are equivalent.

(i) $T$ is injective with closed range. 
(ii) $T$ is $\phi_{+}$with $i(T) \leq 0$ and the limit $\lim _{S \rightarrow 0}(\alpha(T)-\alpha(T-S))$ exists.

(iii) $T$ is $\phi_{+}$with $i(T) \leq 0$ and there is a $\delta>0$ such that for every bounded operator $S: X \rightarrow Y$ with $\|S\|<\delta$ one has $\alpha(T)=\alpha(T-S)$.

(iv) $T \in \operatorname{int}\{$ injective operators $\}$, where $\operatorname{int}\{M\}$ denotes the interior of the set $M$.

Theorem $4[9,2.7]$. Let $X$ and $Y$ be Banach spaces and let $T: X \rightarrow Y$ be a bounded operator. The following conditions are equivalent.

(i) $T$ is surjective.

(ii) $T$ is $\phi_{-}$with $i(T) \geq 0$, and the limit $\lim _{S \rightarrow 0}(\beta(T)-\beta(T-S))$ exists.

(iii) $T$ is $\phi_{-}$with $i(T) \geq 0$ and there exists a $\delta>0$ such that for every bounded operator $S: X \rightarrow Y$ with $\|S\|<\delta$ we have $\beta(T)=\beta(T-S)$.

(iv) $T \in \operatorname{int}\{$ operators with dense range\}.

The purpose of this paper is to obtain characterisations analogous to those of Theorems 3 and 4 in the more general setting of linear relations in normed spaces. The main results of Section 2 (Theorems 7 and 11 below) show that the nullity (respectively, the deficiency) of an almost upper semi-Fredholm (respectively, almost lower semiFredholm) linear relation $T$ remains constant under small perturbations when $T$ is injective and open (respectively, $T$ is almost open with dense range).

\section{Characterisations.}

Proposition 5. Let $T \in \operatorname{AUSF}(X, Y)$ with $\vec{\imath}(T) \leq 0$. Then, for every $\epsilon>0$ there exists $T_{\epsilon} \in L R(X, Y)$ such that $T_{\epsilon}$ is injective and $\left\|T_{\epsilon}-T\right\| \leq \epsilon$.

Proof. Observe that as $\gamma(T)>0$ and $N(T)$ is closed, we have $\gamma(T)=\gamma\left(Q_{T} T\right)$ and $N(T)=N\left(Q_{T} T\right)$ by virtue of [5, II.3.9]. Also, it is clear that $R(T)+\overline{T(0)} \subset \overline{R(T)}$ and $R\left(Q_{T} T\right)=(R(T)+\overline{T(0))} / \overline{T(0)}$ and thus

$$
\begin{aligned}
\beta\left(Q_{T} T\right) & :=\operatorname{dim}(Y / \overline{T(0)}) /(R(T)+\overline{T(0)} / \overline{T(0)})=\operatorname{dim} Y / R(T)+\overline{T(0)} \\
& \geq \operatorname{dim}(Y / R(T)+\overline{T(0)}) /(\overline{R(T)} / R(T)+\overline{T(0)})=\operatorname{dim} Y / \overline{R(T)}:=\bar{\beta}(T) .
\end{aligned}
$$

In consequence, $Q_{T} T$ is an almost upper semi-Fredholm operator such that $N(T)=N\left(Q_{T} T\right)$ and $\alpha(T) \leq \beta\left(Q_{T} T\right)($ as $\overline{1}(T) \leq 0)$.

Let $\left\{x_{1}, x_{2}, \ldots, x_{n}\right\}$ be a basis of $N(T)$ and let $\left[z_{1}\right],\left[z_{2}\right], \ldots,\left[z_{n}\right]$ be linearly independent elements of $Y / \overline{T(0)}$ such that $\left\|x_{i}\right\|=\left\|\left[z_{i}\right]\right\|=1,1 \leq i \leq n$, and $R\left(Q_{T} T\right) \cap$ $\operatorname{span}\left\{\left[z_{1}\right],\left[z_{2}\right], \ldots,\left[z_{n}\right]\right\}=\{[0]\}$. By the Hahn-Banach Theorem, we may choose $\left\{x_{1}^{\prime}, x_{2}^{\prime}, \ldots, x_{n}^{\prime}\right\}$ in $X^{\prime}$ such that $\left\|x_{i}^{\prime}\right\|=1$ and $x_{i}^{\prime}\left(x_{j}\right)=\delta_{i j}, 1 \leq i, j \leq n$. Since $Q_{T}$ is a surjective operator with $Q_{T} B_{Y}=B_{Q_{T} Y}$ there is a set $\left\{z_{1}, z_{2}, \ldots, z_{n}\right\}$ of linearly independent elements of $Y$ such that $Q_{T} z_{i}=\left[z_{i}\right]$ and $\left\|z_{i}\right\| \leq 1,1 \leq i \leq n$.

Let $\epsilon>0$. Define $F_{\epsilon}: x \in X \rightarrow F_{\epsilon} x:=\epsilon / n \sum_{i=1}^{n} x_{i}^{\prime}(x) z_{i} \in Y$.

Then, it is obvious that $F_{\epsilon}$ is a bounded finite rank operator with $\left\|F_{\epsilon}\right\| \leq \epsilon$. We shall verify that $T_{\epsilon}:=T+F_{\epsilon}$ is an injective linear relation and $\left\|T_{\epsilon}-T\right\| \leq \epsilon$.

Since $T(0)=\left(T+F_{\epsilon}\right)(0)=\left(T_{\epsilon}-T\right)(0)$, it follows that $Q_{T}=Q_{T_{\epsilon}}=Q_{T_{\epsilon}-T}$ and so $Q_{T+F_{\epsilon}}\left(T+F_{\epsilon}\right)=Q_{T}\left(T+F_{\epsilon}\right)=Q_{T} T+Q_{T} F_{\epsilon}$. Consequently, $\left\|T_{\epsilon}-T\right\|:=$ $\left\|Q_{T_{\epsilon}-T}\left(T_{\epsilon}-T\right)\right\|=\left\|Q_{T} F_{\epsilon}\right\| \leq \epsilon$.

Finally, since $N\left(T+F_{\epsilon}\right) \subset N\left(Q_{T+F_{\epsilon}}\left(T+F_{\epsilon}\right)\right)=N\left(Q_{T} T+Q_{T} F_{\epsilon}\right)$, to establish the injectivity of $T+F_{\epsilon}$ it is enough to prove that $Q_{T} T+Q_{T} F_{\epsilon}$ is an injective operator. But this last property is trivially true since $\left(Q_{T} T+Q_{T} F_{\epsilon}\right) x=0 \Rightarrow Q_{T} F_{\epsilon} x=-Q_{T} T x \Rightarrow$ $Q_{T} F_{\epsilon} x=0=Q_{T} T x\left(\right.$ as $\left.R\left(Q_{T} T\right) \cap \operatorname{span}\left\{\left[z_{1}\right],\left[z_{2}\right], \ldots,\left[z_{n}\right]\right\}=\{[0]\}\right) \Rightarrow x=0$.

In order to obtain the following Theorem we recall the notion of "graph operator" which can be used to reduce $T \in L R(X, Y)$ to a bounded linear relation. 
Definition 6 [5, IV.3.1]. Given $T \in L R(X, Y)$, let $X_{T}$ denote the vector space $D(T)$ normed by $\|x\|_{T}:=\|x\|+\|T x\|, x \in D(T)$. The graph operator, $G_{T} \in L R\left(X_{T}, X\right)$ is defined by $D\left(G_{T}\right):=X_{T}, G_{T} x:=x$ for $x \in X_{T}$.

In [5, IV.3.2], Cross proves that $\left\|T G_{T}\right\|=\|T\| / 1+\|T\|($ where $\infty / \infty:=1)$. In particular, $T G_{T}$ is a bounded linear relation.

THEOREM 7. Let $T \in L R(X, Y)$. The following properties are equivalent.

(i) $T$ is injective and open.

(ii) $T \in A U S F(X, Y)$ with $\bar{\imath}(T) \leq 0$ and there exists the limit $\lim (\alpha(T)-\alpha(T-S))$ as $S \in L R(X, Y)$ is continuous such that $D(T) \subset D(S), S(0) \subset \overline{T(0)}$ and $S \rightarrow 0$.

(iii) $T \in A U S F(X, Y)$ with $\bar{\imath}(T) \leq 0$ and there is a $\delta>0$ such that for every $S \in$ $L R(X, Y)$ with $D(T) \subset D(S), S(0) \subset \overline{T(0)}$ and $\|S\|<\delta$, we have $\alpha(T)=\alpha(T-S)$.

(iv) There exists a $\delta>0$ such that for every $S \in L R(X, Y)$ with $D(T) \subset$ $D(S), S(0) \subset \overline{T(0)}$ and $\|S\|<\delta$, we have that $T-S$ is injective.

Proof. (i) $\Rightarrow$ (ii) Let $\epsilon>0$ and $0<\delta<\min \{\epsilon, \gamma(T)\}$. Then, if $S$ satisfies $D(T) \subset$ $D(S), S(0) \subset \overline{T(0)}$ and $\|S\|<\delta$, we have by [5, III.7.4] that $\alpha(T-S) \leq \alpha(T)$ and hence $0=\alpha(T)-\alpha(T-S)<\epsilon$, as desired.

(ii) $\Rightarrow$ (iii) We first prove the assertion for the case when $T$ is bounded. By the definition of the limit, there are $\delta>0$ and $d \in \mathbb{N}$ such that for every $S \in B(X, Y)$ with $S(0) \subset \overline{T(0)}$ and $\|S\|<\delta$ one has $0 \leq \alpha(T)-\alpha(T-S)-d<1$. Hence $\alpha(T-S)=$ $\alpha(T)-d$ for all $S \in B(X, Y)$ with $S(0) \subset \overline{T(0)}$ and $\|S\|<\delta$. If $S:=(\delta / 2\|T\|) T$, then it is clear that $S(0)=T(0),\|S\|<\delta$ and thus $\alpha((1-\delta / 2\|T\|) T)=\alpha(T)-d$, and consequently, $d=0$. Therefore $\alpha(T)=\alpha(T-S)$ for all $S \in B(X, Y)$ such that $S(0) \subset \overline{T(0)}$ and $\|S\|<\delta$, as required.

Passing to the general case, we consider the bounded linear relation $T G_{T}$. Then we have $R(T)=R\left(T G_{T}\right), \alpha(T)=\alpha\left(T G_{T}\right)\left(\left[5\right.\right.$, I.6.11]), and $\gamma\left(T G_{T}\right)=\gamma(T) / 1+\gamma(T)([\mathbf{5}$, IV.3.10]), and so if $T$ satisfies (ii), $T G_{T}$ also satisfies (ii) and by what has already been proved, $T G_{T}$ satisfies the condition (iii). Now, let $S \in L R(X, Y)$ such that $D(T) \subset D(S)$, $S(0) \subset \overline{T(0)}$ and $\|S\|<\delta$. (This $\delta$ is obtained in the property (iii) applied to $T G_{T}$.) Therefore, applying (iii) to $T G_{T}$ we obtain $\alpha\left(T G_{T}\right)=\alpha\left((T-S) G_{T}\right)$ and hence $\alpha(T)=$ $\alpha(T-S)$.

(iii) $\Rightarrow$ (iv) By Proposition 5, for every $\epsilon>0$ there is an element $T_{\epsilon} \in L R(X, Y)$ that is injective such that $T_{\epsilon}(0)=T(0), D(T)=D\left(T_{\epsilon}\right)$ and $\left\|T_{\epsilon}-T\right\| \leq \epsilon$. Let $\epsilon=\delta / 2$ and $S_{\epsilon}:=T-T_{\epsilon}$. Then it is obvious that $D(T)=D\left(S_{\epsilon}\right), S_{\epsilon}(0) \subset \overline{T(0)}$ and $\left\|S_{\epsilon}\right\|<\delta$, so that we have $\alpha(T)=\alpha\left(T-S_{\epsilon}\right)=\alpha\left(T_{\epsilon}\right)=0$. Thus for every $S \in L R(X, Y)$ with $D(T) \subset D(S), S(0) \subset \overline{T(0)}$ and $\|S\|<\delta$, we obtain $\alpha(T-S)=0$; that is, the condition (iv) is true.

(iv) $\Rightarrow$ (i) Suppose that $T$ is not injective and open. Then there exists a sequence $\left(x_{n}\right)$ in $X$ for which $\left\|x_{n}\right\|=1$ and $\left\|T x_{n}\right\| \rightarrow 0$ as $n \rightarrow \infty$. By the Hahn-Banach Theorem let $\left(x_{n}^{\prime}\right)$ be a sequence in $X^{\prime}$ such that $1=x_{n}^{\prime}\left(x_{n}\right)=\left\|x_{n}^{\prime}\right\|,(n \in \mathbb{N})$.

For each $n \in N$, we define $T_{n} \in L R(X, Y)$ by $T_{n} x:=x_{n}^{\prime}(x) T x_{n}, x \in X$. Then $T_{n} x_{n}=T x_{n}$ and thus $\left(T_{n}-T\right)(0)=T(0)=T x_{n}-T x_{n}=T_{n} x_{n}-T x_{n}=\left(T_{n}-T\right) x_{n}$; that is, $x_{n} \in N\left(T_{n}-T\right)$. Moreover, since $\left\|T-\left(T-T_{n}\right)\right\| \leq\left\|Q_{T} T x_{n}\right\| \rightarrow 0$ as $n \rightarrow \infty$, we have the implication (iv) $\Rightarrow$ (i).

Corollary 8. Let $T \in L R(X, Y)$ be closed, where $X$ and $Y$ are complete. The following properties are equivalent. 
(i) $T$ is injective with closed range.

(ii) $T$ is $\phi_{+}$with $i(T) \leq 0$ and there exists the limit, $\lim (\alpha(T)-\alpha(T-S))$ as $S \in$ $L R(X, Y)$ is continuous with $D(T) \subset D(S), S(0) \subset \overline{T(0)}$ and $S \rightarrow 0$.

(iii) $T$ is $\phi_{+}$with $i(T) \leq 0$ and there is a $\delta>0$ such that for every $S \in L R(X, Y)$ with $D(T) \subset D(S), S(0) \subset \overline{T(0)}$ and $\|S\|<\delta$, we have $\alpha(T)=\alpha(T-S)$.

(iv) There exists a $\delta>0$ such that for every $S \in L R(X, Y)$ with $D(T) \subset D(S)$, $S(0) \subset \overline{T(0)}$ and $\|S\|<\delta$ one has $T-S$ injective.

Proof. This result follows immediately from Proposition 2 and Theorem 7.

For the particular case of bounded operators the above Corollary coincides with the Theorem 3.

LeMma 9. Let $T \in L R(X, Y)$.

(i) $\alpha(T) \leq \bar{\beta}\left(T^{\prime}\right)$ and $\alpha\left(T^{\prime}\right)=\bar{\beta}(T)$. In particular, if $T$ is $A L S F$ with $\bar{\imath}(T) \geq 0$, then $T^{\prime}$ is AUSF with closed range and $i\left(T^{\prime}\right) \leq 0$.

(ii) Let $S \in L R(X, Y)$ be continuous. Then

(ii $\left.{ }_{1}\right) S^{\prime}$ is continuous and $\|S\|=\left\|S^{\prime}\right\|$.

(iii $)$ If $D(T) \subset D(S)$, then $S^{\prime}(0) \subset \overline{T^{\prime}(0)}$ and $(T+S)^{\prime}=T^{\prime}+S^{\prime}$.

(ii $\left.i_{3}\right)$ If $S(0) \subset \overline{T(0)}$, then $D\left(T^{\prime}\right) \subset D\left(S^{\prime}\right)$.

Proof. (i) We have $N(T) \subset R\left(T^{\prime}\right)^{\top}$. Indeed, $x \in N(T) \Leftrightarrow(x, 0) \in G(T) \subset$ $\overline{G(T)}:=G(\bar{T}) \Leftrightarrow x \in N(\bar{T})$ and $N(\bar{T})=R\left(T^{\prime}\right)^{\top}$ by [5, III.1.4(c]. Therefore $\alpha(T) \leq$ $\bar{\beta}\left(T^{\prime}\right)$. Since $N\left(T^{\prime}\right)=R(T)^{\perp}$ by [5, III.1.4(a)] is $\alpha\left(T^{\prime}\right)=\bar{\beta}(T)$. Moroever, noting that the conjugate of a linear relation is always a closed linear relation we have by [5, III.5.2 and III.5.3] that $T$ is almost open if and only if $T^{\prime}$ is open and $T^{\prime}$ open $\Rightarrow R\left(T^{\prime}\right)$ closed.

(ii) If $S$ is continuous, then so is $S^{\prime}$ and $\|S\|=\left\|S^{\prime}\right\|$ by virtue of [5, III.4.6(c)].

Suppose that $S$ is continuous with $D(T) \subset D(S)$. Then $S^{\prime}(0)=D(S)^{\perp} \subset D(T)^{\perp}=$ $T^{\prime}(0)$ by [5, III. 1.4(b)] and that $(T+S)^{\prime}=T^{\prime}+S^{\prime}$ follows immediately from [5, III.1.5(b)].

Finally, if $S \in L R(X, Y)$ is continuous with $S(0) \subset \overline{T(0)}$, then since $\overline{T(0)} \subset \bar{T}(0)=D\left(T^{\prime}\right)^{\top}$ by [5, II.5.19 and III.1.4(d)], it follows that $D\left(T^{\prime}\right) \subset$ $D\left(T^{\prime}\right)^{\top \perp} \subset \overline{T(0)}{ }^{\perp} \subset S(0)^{\perp}=D\left(S^{\prime}\right)$, where the last equality is deduced by the continuity of $S$ (see [5, III.4.6(a)]. Hence the result follows.

Proposition 10. Let $T \in A L S F(X, Y)$ with $\bar{\imath}(T) \geq 0$. Then, for every $\epsilon>0$ there exists $T_{\epsilon} \in L R(X, Y)$ that is almost open with dense range and $\left\|T_{\epsilon}-T\right\| \leq \epsilon$.

Proof. From Lemma 9 (i) we have that $T^{\prime} \in A U S F\left(Y^{\prime}, X^{\prime}\right)$ such that $R\left(T^{\prime}\right)$ is closed and $i\left(T^{\prime}\right) \leq 0$. Moreover, as $R\left(T^{\prime}\right)$ is closed so is $R\left(Q_{T^{\prime}} T^{\prime}\right)$ and $\beta\left(T^{\prime}\right)=\beta\left(Q_{T^{\prime}} T^{\prime}\right)$. Indeed, as $T^{\prime}$ is a closed linear relation $T^{\prime}(0)$ is a closed subspace of $X^{\prime}$ by [5, II.5.3 and III.1.2] and hence $R\left(Q_{T^{\prime}} T^{\prime}\right)=R\left(T^{\prime}\right) / T^{\prime}(0)$ is closed in $X^{\prime} / T^{\prime}(0)$ and $\beta\left(Q_{T^{\prime}} T^{\prime}\right):=\operatorname{dim}\left(X^{\prime} / T^{\prime}(0)\right) /\left(R\left(T^{\prime}\right) / T^{\prime}(0)\right)=\operatorname{dim} X^{\prime} / R\left(T^{\prime}\right):=\beta\left(T^{\prime}\right)$ by virtue of [5, IV.5.2].

Let $\left\{y_{1}^{\prime}, y_{2}^{\prime}, \ldots, y_{n}^{\prime}\right\}$ be a basis of $N\left(T^{\prime}\right)=N\left(Q_{T^{\prime}} T^{\prime}\right)$ and we can find a set $\left\{\left[z_{1}^{\prime}\right],\left[z_{2}^{\prime}\right], \ldots,\left[z_{n}^{\prime}\right]\right\}$ of linearly independent elements of $Q_{T^{\prime}} X^{\prime}$ (so that $\left[z_{i}^{\prime}\right]=Q_{T^{\prime}} x_{i}^{\prime}$ with $\left\{x_{1}^{\prime}, x_{2}^{\prime}, \ldots, x_{n}^{\prime}\right\}$ linearly independent) such that $\left\|y_{i}^{\prime}\right\|=\left\|\left[z_{i}^{\prime}\right]\right\|=1, R\left(Q_{T^{\prime}} T^{\prime}\right) \cap$ $\operatorname{sp}\left\{\left[z_{1}^{\prime}\right],\left[z_{2}^{\prime}\right], \ldots,\left[z_{n}^{\prime}\right]\right\}=\{[0]\}$ and $\left\|x_{i}^{\prime}\right\| \leq 1$. Let $y_{1}, y_{2}, \ldots, y_{n}$ be chosen in $Y$ such that $\left\|y_{i}\right\|=1$ and $y_{i}^{\prime}\left(y_{j}\right)=\delta_{i j}$. 
Now define for $\epsilon>0$ the operator $F_{\epsilon} \in L R(X, Y)$ as follows:

$$
F_{\epsilon}(x):=\epsilon / n \sum_{i=1}^{n} x_{i}^{\prime}(x) y_{i}(x \in X) .
$$

Observe that $F_{\epsilon}$ is a bounded finite rank operator such that its norm does not exceed $\epsilon$ and its conjugate $F_{\epsilon}^{\prime}$ is defined by $F_{\epsilon}^{\prime} y^{\prime}=\epsilon / n \sum_{i=1}^{n} y^{\prime}\left(y_{i}\right) x_{i}^{\prime}, y^{\prime} \in Y^{\prime}$.

We shall verify that $T_{\epsilon}:=T+F_{\epsilon}$ is almost open with dense range and $\left\|T_{\epsilon}-T\right\| \leq$ $\epsilon$. Since $\gamma\left(T^{\prime}\right)>0, \bar{\beta}(T)<\infty$ and $F_{\epsilon}$ is a bounded finite rank operator, it follows from [5, V.5.2 and V.5.12] that $T_{\epsilon}$ is almost open. Upon observing that $R\left(Q_{T^{\prime}} T^{\prime}\right) \cap$ $R\left(Q_{T^{\prime}} F_{\epsilon}^{\prime}\right)=\{[0]\},\left(T+F_{\epsilon}\right)^{\prime}=T^{\prime}+F_{\epsilon}^{\prime}$ (by Lemma 9 (ii)) and proceeding exactly as in the proof of Proposition 5 we deduce that $\left(T+F_{\epsilon}\right)^{\prime}$ is injective (equivalently $T+F_{\epsilon}$ has dense range) and $\left\|T_{\epsilon}-T\right\| \leq \epsilon$, as required.

THEOREM 11. Let $T \in L R(X, Y)$. The following conditions are equivalent.

(i) $T$ is almost open with dense range.

(ii) $T$ is $A L S F$ with $\bar{\imath}(T) \geq 0$ and there exists the limit $\lim (\bar{\beta}(T)-\bar{\beta}(T-S))$ as $S \in L R(X, Y)$ is continuous with $D(T) \subset D(S)$ and $S(0) \subset \overline{T(0)}$.

(iii) $T$ is $A L S F$ with $\bar{l}(T) \geq 0$ and there is a $\delta>0$ such that, for every $S \in L R(X, Y)$ with $D(T) \subset D(S), S(0) \subset \overline{T(0)}$ and $\|S\|<\delta$ we have $\bar{\beta}(T)=\bar{\beta}(T-S)$.

(iv) There exists a $\delta>0$ such that for every $S \in L R(X, Y)$ with $D(T) \subset D(S)$, $S(0) \subset \overline{T(0)}$ and $\|S\|<\delta, T-S$ has dense range.

Proof. (i) $\Rightarrow$ (ii) Assume that (i) holds. Let $\epsilon>0$ and $0<\delta<\min \left\{\epsilon, \gamma\left(T^{\prime}\right)\right\}$. Then, for every $S \in L R(X, Y)$ with $D(T) \subset D(S), S(0) \subset \overline{T(0)}$ and $\|S\|<\delta$ we conclude from Lemma 9 and [5, III.7.4] that $0=\bar{\beta}(T)-\bar{\beta}(T-S)<\epsilon$.

(ii) $\Rightarrow$ (iii) This implication is an immediate consequence of (ii) $\Rightarrow$ (iii) of Theorem 7 using Lemma 9.

(iii) $\Rightarrow$ (iv) By Proposition 10, for every $\epsilon>0$ there exists $T_{\epsilon} \in L R(X, Y)$ such that $D(T)=D\left(T_{\epsilon}\right), T_{\epsilon}(0)=T(0)$ and $\left\|T_{\epsilon}-T\right\| \leq \epsilon$. Let $\epsilon=\delta / 2$ and $S_{\epsilon}:=T-T_{\epsilon}$. Then it is easy to show that $S_{\epsilon}(0) \subset \overline{T(0)}, D(T) \subset D\left(S_{\epsilon}\right)$ and $\left\|S_{\epsilon}\right\|<\delta$ and so we have $\bar{\beta}(T)=\bar{\beta}\left(T-S_{\epsilon}\right)=\bar{\beta}\left(T_{\epsilon}\right)=0$. Thus for every $S \in L R(X, Y)$ with $D(T) \subset D(S)$, $S(0) \subset \overline{T(0)}$ and $\|S\|<\delta$ we obtain $\bar{\beta}(T-S)=0$, as desired.

(iv) $\Rightarrow$ (i) Suppose that (i) does not hold. Then $T^{\prime}$ is not injective and open and thus there are a pair of sequences $\left(y_{n}^{\prime}\right)$ in $D\left(T^{\prime}\right)$ and $\left(y_{n}\right)$ in $Y$ such that $1=y_{n}^{\prime}\left(y_{n}\right)$ and $\left\|T^{\prime} y_{n}^{\prime}\right\| \rightarrow 0$ as $n \rightarrow \infty$. We define $T_{n} \in L R(X, Y)$ by

$$
T_{n} x:=T^{\prime} y_{n}^{\prime}(x) y_{n}, x \in X .
$$

Then $T_{n}^{\prime} y^{\prime}=y^{\prime}\left(y_{n}\right) T^{\prime} y_{n}^{\prime}$ for $y^{\prime} \in Y^{\prime}$ and hence $T^{\prime} y_{n}^{\prime}=T_{n}^{\prime} y_{n}^{\prime}$. Arguing as in the last paragraph of the proof of Theorem 7 (iv) $\Rightarrow$ (i), we obtain that $y_{n}^{\prime} \in N\left(\left(T-T_{n}\right)^{\prime}\right)=$ $N\left(T^{\prime}-T_{n}^{\prime}\right)$ (this equality is true by Lemma 9(ii)), so that $\left(T-T_{n}\right)^{\prime}$ is not injective; that is, $R\left(T-T_{n}\right)$ is not dense. Furthermore, it is obvious that $\left\|T-\left(T-T_{n}\right)\right\| \leq\left\|T^{\prime} y_{n}^{\prime}\right\| \rightarrow$ 0 as $n \rightarrow \infty$, contradicting (iv). Therefore (iv) $\Rightarrow$ (i) as required.

Corollary 12. Let $T \in L R(X, Y)$ be closed where $X$ and $Y$ are Banach spaces. The following properties are equivalent.

(i) $T$ is surjective.

(ii) $T$ is $\phi_{-}$with $i(T) \geq 0$, and there exists the limit $\lim (\beta(T)-\beta(T-S))$ as $S \in$ $L R(X, Y)$ is continuous with $D(T) \subset D(S), S(0) \subset \overline{T(0)}$ and $S \rightarrow 0$. 
(iii) $T$ is $\phi_{-}$with $i(T) \geq 0$ and there exists a $\delta>0$ such that for every $S \in L R(X, Y)$ with $D(T) \subset D(S), S(0) \subset \overline{T(0)}$ and $\|S\|<\delta$ we have $\beta(T)=\beta(T-S)$.

(iv) There exists $\delta>0$ such that for every $S \in L R(X, Y)$ with $D(T) \subset D(S)$, $S(0) \subset \overline{T(0)}$ and $\|S\|<\delta$ one has $R(T-S)$ dense.

Proof. Apply Proposition 2 with Theorem 11.

For the particular case of bounded operators the above Corollary coincides with Theorem 4.

ACKNOWLEDGEMENT. The author would like to thank the referee for some helpful comments.

\section{REFERENCES}

1. J. P. Aubin and A. Cellina, Differential inclusions (Springer-Verlag, 1984).

2. F. H. Clarke, Optimization and nonsmooth analysis (Wiley-Interscience, 1983).

3. E. A. Coddington, Multivalued operators and boundary value problems, Lecture Notes in Mathematics, No. 183, (Springer-Verlag, 1971).

4. E. A. Coddington and A. Dijksma, Selfadjoint subspaces and eigenfunction expansions for ordinary differential subspaces, J. Differential Equations 20 (1976), 473-526.

5. R. W. Cross, Multivalued linear operators, Monographs and Textbooks in Pure and Applied Mathematics 213 (Marcel Dekker, 1998).

6. A. Favini and A. Yagi, Multivalued linear operators and degenerate evolution equations, Ann. Mat. Pura. Appl. (4) 163 (1993), 353-384.

7. M. Gromov, Partial differential relations (Springer-Verlag, 1986).

8. R. Harte, Invertibility and singularity for bounded linear operators (Marcel Dekker, 1988). 191-201.

9. M. Mbekhta, On the stability of semi-Fredholm operators, J. Operator Theory 35 (1996),

10. J. von Neumann, Functional operators, Vol. 2. The geometry of orthogonal spaces, Ann. Math. Stud., 22 (Princeton University Press, 1950). 